\title{
Alterations in spatial learning and memory after forced exercise
}

\author{
Eng-Tat Ang ${ }^{a, d, *}$, Gavin S. Dawe ${ }^{b}$, Peter T.H. Wong ${ }^{b}$, \\ Shabbir Moochhala ${ }^{b, c}$, Yee-Kong $\mathrm{Ng}^{a, *}$ \\ ${ }^{a}$ Department of Anatomy, National University of Singapore, Singapore \\ ${ }^{\mathrm{b}}$ Department of Pharmacology, National University of Singapore, Singapore \\ ${ }^{\mathrm{c} D e f e n c e}$ Medical and Environmental Research Institute, DSO National Laboratories (Kent Ridge), Singapore \\ ${ }^{\mathrm{d}}$ Institute of Histology and General Embryology, University of Fribourg, Switzerland
}

\begin{abstract}
A B S T R A C T
Exercise has been shown to influence learning and memory. Most studies were performed with a voluntary running paradigm (e.g. running wheel) in mice. However, such effects of exercise on learning and memory are less well demonstrated using a forced running paradigm (e.g. treadmill). The present study was designed to examine the effects of 12 weeks of forced treadmill running on learning and memory performance in rats. We have previously shown that forced running resulted in qualitative and quantitative changes in the cholinergic neurons of the horizontal diagonal band of Broca (HDB) in the septum. This study was conducted in order to determine whether or not these changes occur simultaneously with enhanced learning and memory. The one-day version of the Morris water maze (MWM) test [Frick, K.M., Stillner, E.T., Berger-Sweeney, J., 2000. Mice are not little rats: species differences in a one-day water maze task. NeuroReport 11, 3461-3465] was used to test spatial learning and memory after the exercise period. Our data showed that runners displayed better spatial learning and memory when compared to nonrunners. This was evidently shown by a reduction in the time required for spatial acquisition $(p<0.05)$ and superior probe trial performance $(p<0.05)$. A shorter distance swam by the runners also suggested improved learning over the nonrunners $(p<0.05)$. In an attempt to revalidate our earlier quantitative results, we used design-based stereology (DBS) to estimate the number of cholinergic neuronal profile population in the medial septum and diagonal band (MSDB). We confirmed that forced running increased the cholinergic neuronal profile subpopulation in the HDB (Coefficient of Error <0.2). Taken together, these results indicate that forced exercise could influence learning and memory with a concomitant increase in the number of cholinergic neurons in the HDB.
\end{abstract}

\footnotetext{
${ }^{*}$ Corresponding authors. E.-T. Ang is to be contacted at Institute of Histology and General Embryology, University of Fribourg, Switzerland. Fax: +41 26300 9732. Y.-K. Ng, Department of Anatomy, Yong Loo Lin School of Medicine, National University of Singapore, MD10, 4 Medical Drive, 117597, Singapore. Fax: +65 67787643.

E-mail address: antngyk@nus.edu.sg (Y.-K. Ng).
} 


\section{Introduction}

It has been reported that learning and memory can be influenced by exercise. Animal studies on rats and mice reported better cognitive performance as a result of increased physical activities (Samorajski et al., 1985; Fordyce and Farrar, 1991a,b; Anderson et al., 2000). In human subjects, it was reported that physically fit individuals have better cognitive and memory performance when compared to their sedentary peers (Diesfeldt and DiesfeldtGroenendijk, 1977; Young, 1979; Emery et al., 1995). In support of better cognitive performance, it appears that exercise could enhance neurogenesis (Van Praag et al., 1999) and up-regulate the expression of trophic factors (Neeper et al., 1996; Ang et al., 2003). However, there exist some controversies in the literature, for example, 6-week long physical exercise has been reported to slow the parallel learning of a complex task in rats (Braszko et al., 2001).

Most studies involving exercise were based on voluntary running paradigms (Samorajski et al., 1985; Neeper et al., 1996; Van Praag et al., 1999; Anderson et al., 2000). While this is largely in line with ethological animal behaviors, voluntary and forced exercise influenced survival and body composition differently in rats (Narath et al., 2001). A forced exercise regime is more akin to human physical training and arguably more objective in terms of outcome measure as it allows for more accurate correlation between the amounts of exercise to any potential benefits arising therefrom. For instance, it was documented that a certain distance must be covered before any biochemical changes were observed (Shen et al., 2001). Fordyce and Farrar (1991a, b) reported that F344 rats exhibited enhanced performance on certain spatial tasks after a 14-week treadmill running program. These runners also showed enhanced hippocampal high-affinity choline uptake and muscarinic receptor binding.

We have previously reported that the number of cholinergic neurons in the HDB, but not in the medial septum nucleus (MSN) and vertical diagonal band (VDB), was increased significantly after forced running (Ang et al., 2003) which supported the view that cholinergic mechanisms modulate learning and memory formation (Gold, 2003). However, our earlier approach did assume some geometric and spatial properties of the cells concerned and therefore might have resulted in bias. It has been reported that results obtained with design-based stereology (DBS) is independent of size, shape, spatial distribution and orientation (assumption-free), and the bias involved can be limited to a minimum (Schmitz and Hof, 2005). We therefore decided to revalidate our results by DBS on the number of cholinergic neurons in the above nuclei since there were no apparent differences between the runner and nonrunner in the MSN and VDB. Hence, the objectives of the present study are: (1) to elucidate any changes in spatial learning and memory in rats after 12 weeks of forced treadmill running and (2) to revalidate the previously reported increase in the number of cholinergic neurons in the HDB after forced running by DBS.

\section{Results}

\subsection{Spatial acquisition and pathlength}

Typical swim-paths of the nonrunner and runner are shown in Figs. $1 \mathrm{~B}$ and $\mathrm{C}$ respectively. All rats showed a reduction in
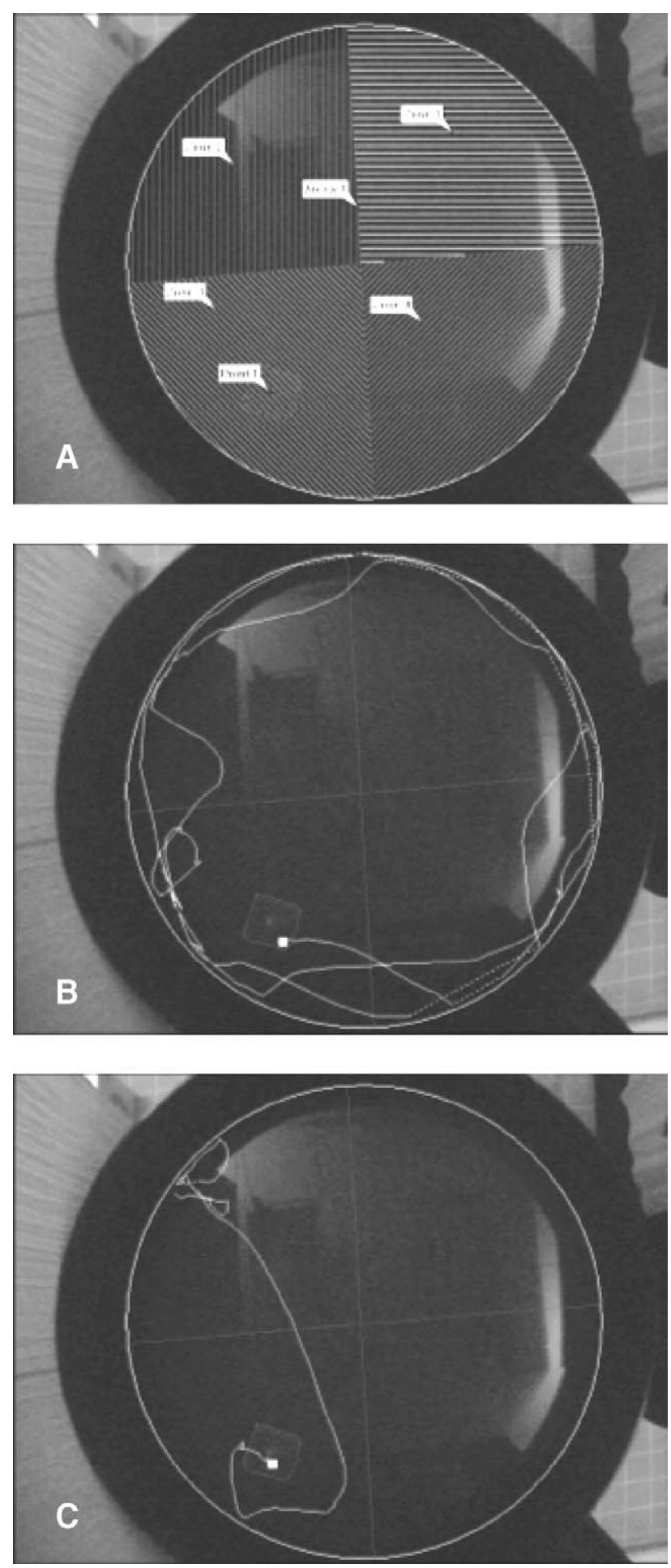

Fig. 1 - (A) Pictures of the water maze setup detailing the four zones. (B) Typical swim path of nonrunner. (C) Typical swim path of runner. 
escape latencies (BLOCK effect, $F(2,124)=767, p<0.001$; see Fig. 2) and a reduction in the distance swam to locate the platform (BLOCK effect, $F(2,124)=7327, p<0.001$; see Fig. 3) across blocks of trials, indicating spatial acquisition. Runners found the platform more quickly than nonrunners $(19.8 \pm 0.2 \mathrm{~s}$ and $27.9 \pm$ $0.2 \mathrm{~s}$, respectively; RUNNING effect, $F(1,62)=1480, p<0.001)$ and took shorter paths to the platform $(4.82 \pm 1.14 \mathrm{~m}$ and $7.83 \pm$ $1.14 \mathrm{~m}$, respectively; RUNNING effect, $F(1,62)=34,846, p<$ 0.001). Across the blocks of trials, the runners improved more rapidly than the nonrunners as indicated by both the escape latencies (RUNNING*BLOCK effect interaction, $F(2,124)=$ 80.23, $p<0.001$; see Fig. 2) and the shorter pathlengths (RUNNING*BLOCK effect interaction, $F(2,124)=776.49, p<$ 0.001; see Fig. 3).

\subsection{Swim speed}

Runners swam slower than nonrunners $\left(0.240 \pm 0.00258 \mathrm{~ms}^{-1}\right.$ and $0.28 \pm 0.00258 \mathrm{~ms}^{-1}$, respectively; RUNNING effect, $F(1,62)=$ 127.85, $p<0.001$; see Fig. 4). Swim speed also decreased across blocks (BLOCK effect, $F(2,124)=83.26, p<0.001$; see Fig. 4), and the pattern of change in swim speed differed between runners and nonrunners (RUNNING*BLOCK effect interaction, $F(2,124)=32.07, p<0.001$; see Fig. 4).

\subsection{Probe trial}

Results from the probe trial as measured by the mean percentage (\%) time spent in each of the four zones indicated that the runners spent more time in zone 3 , where the platform was previously located, than the nonrunners $(33.9 \pm 0.5 \%$ and $29.4 \pm 0.2 \%$, respectively; $F(1,15)=555.98,{ }^{*} p<0.001$; see Fig. 5). Nonrunners spent more time in the adjacent zone 4 than runners $(26.7 \pm 1.2 \%$ and $22.4 \pm 2.1 \%$, respectively; $F(1,15)=$ 24.97, $\# p<0.001$; see Fig. 5).

\subsection{Profile of ChAT neurons and stereological details}

Under low magnification, cholinergic neurons were distributed in the MSN, VDB, and HDB of the septum at all time points

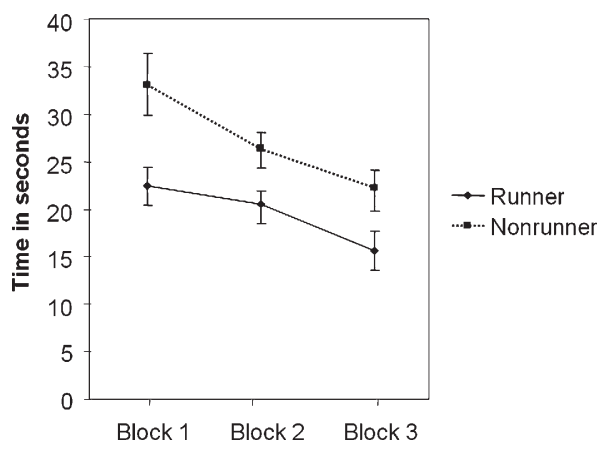

Fig. 2 - Effects of forced exercise on escape latencies to reach the platform. Each point represents the block mean ( \pm SEM) of 4 swims. For latency, lower numbers indicate better performance. Runner $(n=8)$ exhibited better performance than the nonrunner $(n=8)$ throughout the experiment as confirmed by two-way ANOVA (running effect and block effect) $(p<0.05)$ (see text for details of the statistics).

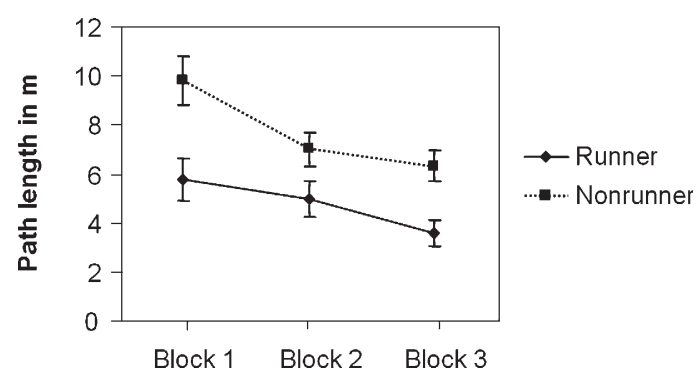

Fig. 3 - Effects of forced exercise on the average pathlength (m). Each point represents the block mean ( \pm SEM) of 4 swims. The runner $(n=8)$ consistently took shorter paths compared to the nonrunner $(n=8)$ as confirmed by two-way ANOVA (running effect and block effect) $(p<0.05)$ (see text for details of the statistics).

(Fig. 6). Fig. 7 shows staining of the cholinergic neurons in the HDB of nonrunners (A and C) and runners (B and D) after the 12th week. At higher magnification, it was observed that the cholinergic neurons (arrows) in the HDB of nonrunners (C) were less numerous and perhaps less intensely stained than those in the HDB of runners (D). More importantly, reevaluation of the cell profile in the HDB using the "Fractionator" probe confirmed that this was significantly increased after 12 weeks of forced running (Fig. 8C). No significant differences were observed in the MSN and VDB (Figs. 8A and $B)$. The coefficients of errors (CE) obtained for analysis were consistently $<0.2$ (see Table 1 for details).

\section{Discussion}

In this study, we report that the forced running paradigm is effective in enhancing spatial learning and memory in rats. At the end of 12 weeks of treadmill running, the runners would have covered an approximate total distance of $90 \mathrm{~km}$, averaging about $1.5 \mathrm{~km} /$ day. This is well above the threshold of $0.5 \mathrm{~km} /$ day previously indicated for activation of signaling molecules that influence synaptic plasticity, including learning and memory (Shen et al., 2001). While the voluntary

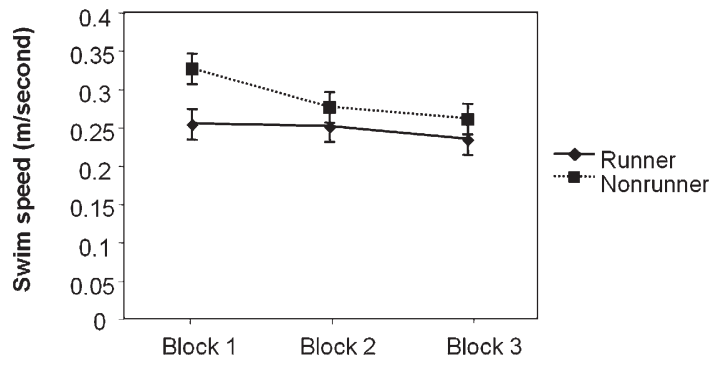

Fig. 4 - Average swim speed of runner $(n=8)$ and nonrunner $(n=8)$ at different block intervals. Each point represents the block mean ( \pm SEM) of 4 swims. Both runners and nonrunners displayed decreasing speed over time as determined by two-way ANOVA (running effect and block effect) $(p<0.05)$ (see text for details of the statistics). 


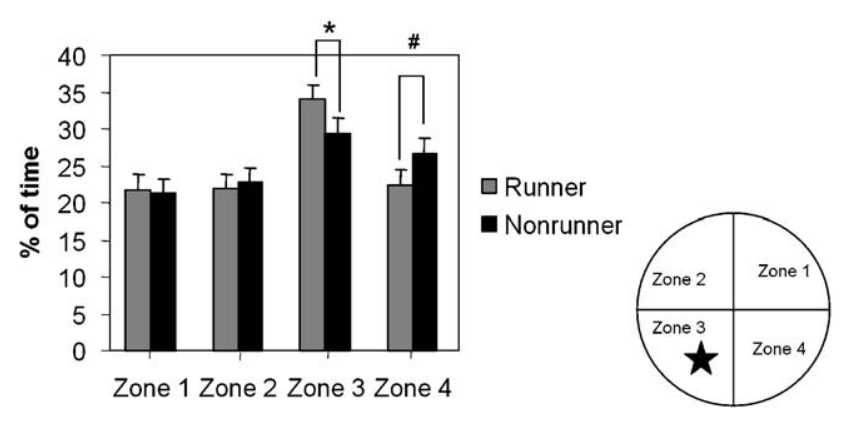

Fig. 5 - Effects of forced exercise on performance during the probe trial as measured by mean percentage (\%) time spent in each of the four zones. Zone 3 with a star is the zone where the platform was previously located. Runner $(n=8)$ spent significantly more time in zone 3 than the nonrunners $(n=8)$ $(p<0.05)$ as determined by multivariate ANOVA. In contrast, nonrunners spent more time in the adjacent zone 4 than runners $(\# p<0.05)$ (see text for statistics).

paradigm (running wheel) also allowed estimation of distance covered, it is felt that the running speed might differ over time. In comparison, the treadmill system therefore offers more accountability compared to the running wheel system.

The forced running paradigm is associated with a certain level of stress (Arida et al., 2004). Rats were forced to run at the speed of the treadmill, otherwise they would receive a mild electric shock from the grid, located just behind the treadmill. The stress associated with the likelihood of getting shock was controlled by exposing the control rats to the treadmill apparatus without switching on the treadmill. These rats would receive the same electric shock when they stepped onto the grid. Nearly all rats learned quickly and rarely received more than a few shocks. Therefore, they were not in chronic stress because they were free to stay on the stationary treadmill to avoid getting shocked. In contrast, the runners were forced to run to avoid being transported to the grid by the moving treadmill. Such acute stress is likely to affect the learning process since it was previously reported that emotionally arousing experiences such as stress often facilitate the formation of memory more readily (Christianson, 1992). Mechanistically, this could be attributed to the release of adrenal stress hormones, epinephrine, and cortisol (corticosterone in the rat), as a consequence of the emotional arousal (McGaugh, 2000; McEwen, 2000b). The elevated stress hormones associated with forced running could be the underlying reason why runners had acquired better memory and learning abilities. Prolonged stress, on the other hand, results in adaptive plasticity in the brain, in which local neurotransmitters as well as systemic hormones interact to produce structural as well as functional changes (McEwen, $2000 b$ ). This includes the suppression of ongoing neurogenesis in the dentate gyrus (Gould et al., 1997; McEwen, 2000a,b).

As a secondary observation, it was found that the runner's body weight was considerably lower compared to the nonrunners. Furthermore, it was also determined that the runners have increased heart mass and larger brain sizes. It was most probable that the strenuous nature of the treadmill running might have resulted in increased metabolic demand, and hence greater heart and brain sizes. These data are in line with previous literature implicating the role of enriched environment and exercise to observable brain changes (Cummins et al., 1973; Anderson et al., 2002). More simplistically, it was indicative of the training effects of treadmill running.

Our present results clearly demonstrated that forced running could significantly improve spatial learning and memory. This conclusion is supported by the superior escape latencies, more time spent in the correct zone, and reduced pathlength in the MWM of the runners over the nonrunners. Our finding that spatial memory is improved by running is consistent with previous reports (Samorajski et al., 1985; Fordyce and Farrar, 1991a,b; Anderson et al., 2000). However, Barnes et al. (1991) did not observe any beneficial effects from exercise, while Braszko et al. (2001) observed that 6 weeks of daily treadmill running has an adverse influence on 'poor performers', but no effects on 'good performers' in learning a complex tasks. Apart from the length of training, such differing outcomes might be accounted for by differences in strain and age of the animals used since these are all known to affect the results (Wyss et al., 2000). In addition, the different behavioral tests, which the rats were subjected to, could also play a role.

Interestingly, the runners swam at a slower average and maximum speed than the nonrunners at all block intervals, although one might have assumed the opposite because of the shorter escape latencies for the runners. It is speculated at this juncture that this might be due to their superior cognitive abilities but fatigue associated with the treadmill running may also have an influence.

In conclusion, there is considerable supportive evidence that exercise, whether forced or voluntary, may indeed help to improve spatial learning and memory. It was reported that exercise could promote long-term potentiation (LTP) (Van Praag et al., 1999). This is significant as it is widely accepted that LTP may serve as a model for some of the molecular and synaptic events underlying memory (Abraham, 2003), including the formation of dendritic spine (Toni et al., 1999). It is also interesting to note that such increased LTP might be the result of increased endogenous neurotrophic factors due to exercise. The use of exogenous neurotrophic factors to help improve spatial reference and recent memory has previously been

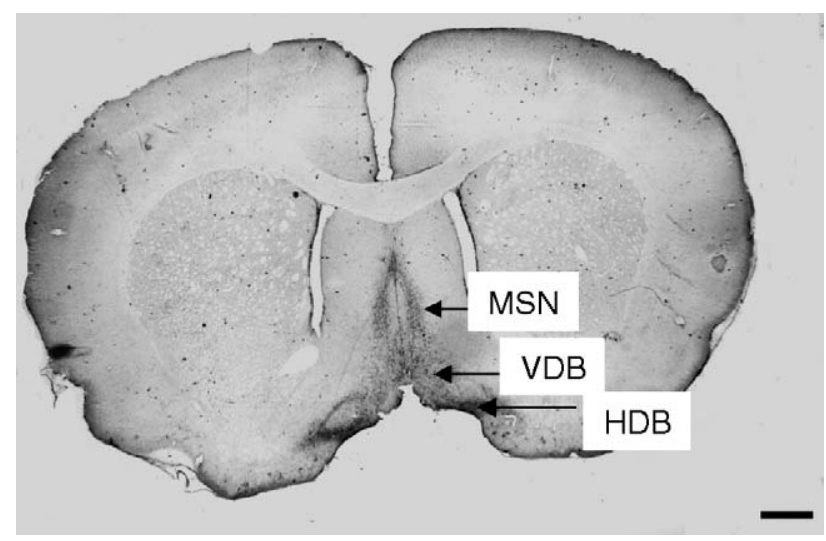

Fig. 6 - Gross distribution of cholinergic neurons in the MSN, VDB, and HDB in the septum of the basal forebrain (Bregma: $1.0 \mathrm{~mm}$ ). Scale bar $=400 \mu \mathrm{m}$. 


\section{Nonrunner}
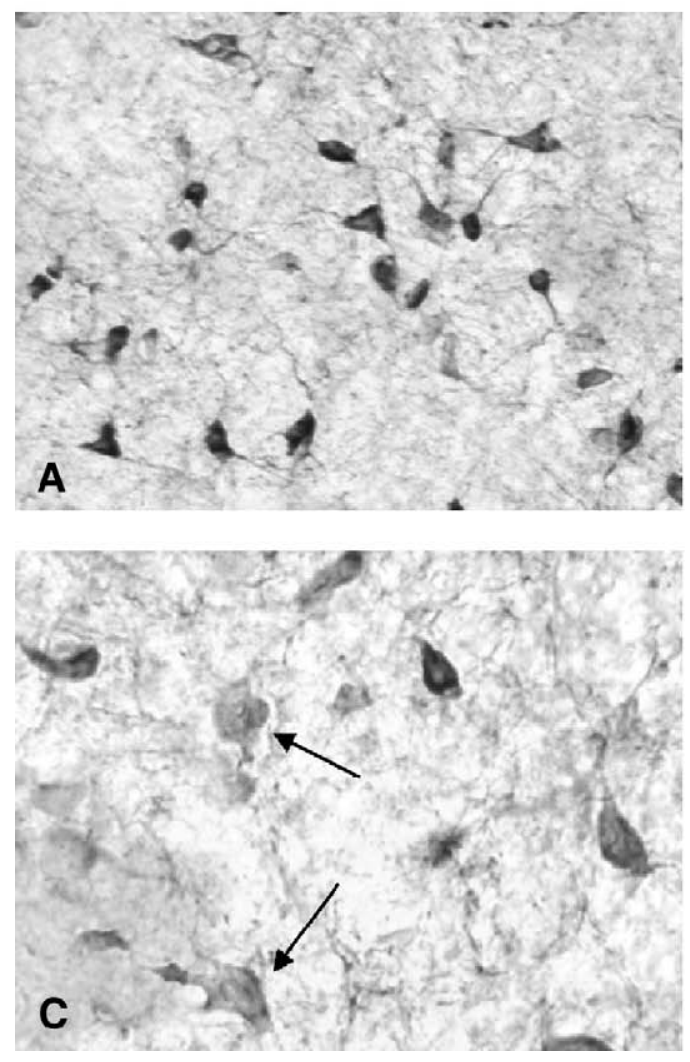

\section{Runner}
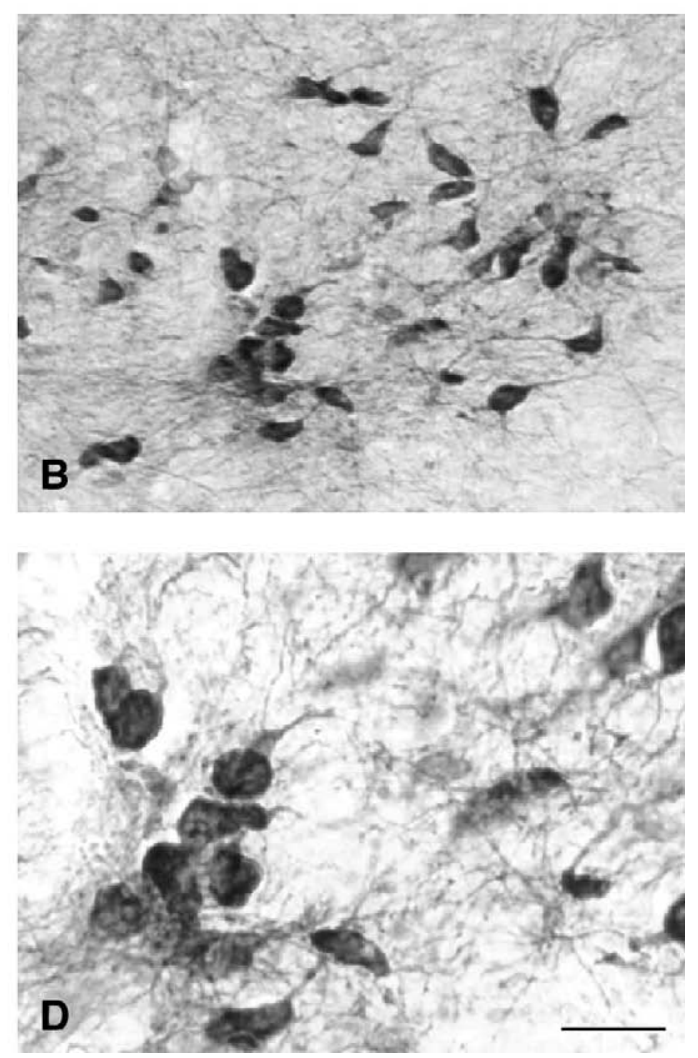

Fig. 7 - Qualitative changes in the cholinergic neurons of the HDB, after 12 weeks of treadmill training in the nonrunner (A) and runner (B). Noticed that there were more ChAT IR neurons in (B) compared to panel A. Furthermore, at higher magnification, the ChAT IR neurons (arrows) in the nonrunner (C) stained less intensely compared to those for the runners (D). Scale bar $=50 \mu \mathrm{m}(A$ and $B)$ and $25 \mu \mathrm{m}(C$ and $D)$.

suggested (Markowska et al., 1996; Frick et al., 1997). LTP could also be moderated by changes in endogenous cytokines such as TNF- $\alpha$ and IL-1 $\beta$ (Cunningham et al., 1996; Butler et al., 2004) as a direct consequence of exercise (Ang et al., 2004). Both TNF$\alpha$ and IL-1 $\beta$ are known to inhibit LTP. We believed that both voluntary and forced exercise could help spatial learning and memory not just in rodents but also in humans. The possibility that exercise has indirectly influenced the brain via hormonal or metabolic changes is indeed very real. However, further research should be conducted to provide more direct evidence. Testing for stress hormones in the blood might help to fill the gap.

\subsection{Methodological consideration}

Previously, we had reported that forced running increased the number of cholinergic cells in the HDB (Ang et al., 2003). In our previous effort, the number of cholinergic neurons counted in the MSDB was guided and corrected by Abercrombie's principle (Abercrombie, 1946). With the advent of computer-aided stereology, we have re-evaluated the number of cholinergic neuronal profile in the MSDB with the greater precision afforded by application of state-of-the-art DBS (Schmitz and Hof, 2005). In the DBS approach, the CE assesses the variability of the results obtained (Schmitz and Hof, 2005). We have confirmed that forced exercise increases the number of cholinergic neuronal profile in the HDB. With this more sensitive and accurate method, we also confirmed that exercise did not result in any changes to the cholinergic neuronal profile subpopulation in the MSN and VDB. We still do not know the reasons why only the HDB was influenced by exercise.

\section{Experimental procedures}

\subsection{Subjects and exercise regime}

Male Wistar rats (200 to $250 \mathrm{~g}$ ) obtained from the Laboratory Animal Center (National University of Singapore) were used in the study. All experiments were carried out in accordance with the National Institute of Health Guide for the Care and Use of Laboratory Animals (NIH Publications No. 80-23) revised 1996. Rats were divided into two age-matched groups. The "runner" $(n=8)$ was subjected to daily treadmill running (Simplex II metabolic rodent treadmill equipped with an electro-stimulator (ES) at the rear, Columbus Instruments) at an inclination of $0^{\circ}$ as we have previously described (Ang et al., 2003). Briefly, in the first week, the treadmill speed was increased from 5 to $30 \mathrm{~m} \mathrm{~min}^{-1}$ over the first $5 \mathrm{~min}$ per run, and the duration was increased from 10 to 50 min per run over 5 days. From week 2 
A

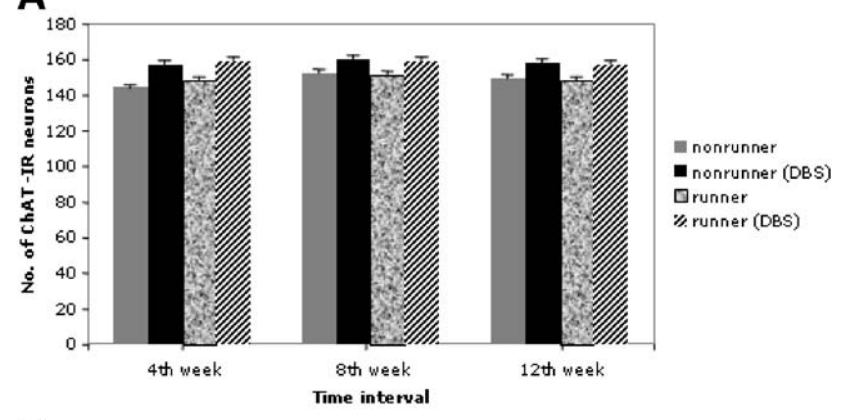

B

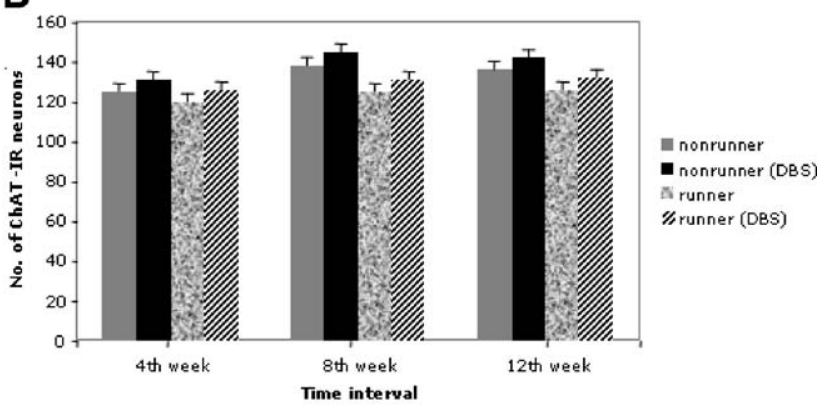

c

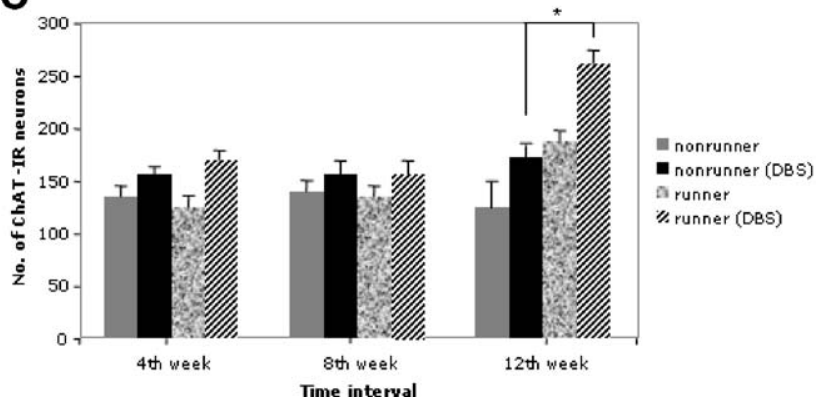

Fig. 8 - Graphs showing the average number of cholinergic neuronal profile in the (A) MSN, (B) VDB, and (C) HDB between the nonrunner and runner, across the different time points. Comparison was made with data obtained earlier (Ang et al., 2003). Using DBS and independent sample $t$ tests, it was concluded that there were more cholinergic neurons in the HDB of the runners compared to the nonrunners after 12 weeks of forced exercise $\left({ }^{*} p<0.05\right)$.

onwards, speed and duration were kept constant at $30 \mathrm{~m} \mathrm{~min}^{-1}$ and 50 min per run. The "nonrunner" $(n=8)$ was always placed in a neighboring lane without switching on the treadmill for the exact duration as the runners but was not forced to run. The ES delivered electric shocks when the rats entered the rear of the test chamber. Both runners and nonrunners could avoid the shocks by remaining on the treadmill. For the runners, remaining on the moving treadmill required the rats to run. Outside of the training schedule, all rats were housed individually in cages and had unlimited access to water and food.

\subsection{Water maze protocol}

The one-day water maze test as described previously (Frick et al., 2000) with minor modifications was carried out on both runners and nonrunners. This version of the water maze test was chosen for practical reasons as it could rapidly evaluate learning and memory in rodents. The setup consisted of a circular tank ( $190 \mathrm{~cm}$ in diameter) and filled with water (up to $30 \mathrm{~cm}$ deep; temperature: $22 \pm 2{ }^{\circ} \mathrm{C}$ ) made opaque by the addition of a non-toxic dye. The tank was further divided into four zones (Fig. 1A). A platform $(18 \mathrm{~cm} \times 18 \mathrm{~cm})$ was submerged $2 \mathrm{~cm}$ below the water surface in zone 3 . The pool was placed in a small room with extra-maze cues, kept constant throughout the experiment. The data were recorded and analyzed with an overhead video-camera connected to a computer running Ethovision Pro software (Version 2.3.19, Noldus). For training, all rats were exposed 4 times to the setup over 2 days. Each rat was given 60 s to randomly explore the water maze, and in this period if it did not find the platform by chance, it would be guided to it and allowed to remain there for $10 \mathrm{~s}$ to familiarize itself with the location of the platform relative to the visual cues in the room. Formal data recording only commenced thereafter.

Any improvements in spatial learning and memory are confirmed by the spatial acquisition and probe trial respectively. In the first test (spatial acquisition), each rat underwent three blocks of 4 swims separated by a 30-minute interval. The starting position for each swim was always randomly chosen. Each rat was given $60 \mathrm{~s}$ to reach the platform, otherwise it would be guided to the platform and allowed to remain there for $10 \mathrm{~s}$. Escape latencies (s), pathlength $(\mathrm{m})$, and the swim speed $(\mathrm{m} / \mathrm{s})$ were recorded. The second test (probe trial) which was conducted after a 30min break involved removing the platform and the rats undergoing a single trial of $60 \mathrm{~s}$. The percentage of time spent in each zone was recorded. After the end of each block, all animals were put back into their cages to rest. Lamps were used to help the animals to stay warm during the experiments.

\subsection{Immunohistochemistry}

All rats from both cohorts were sacrificed, and their brains harvested as described in our earlier study (Ang et al., 2003). Briefly, rats were anaesthetized with $7 \%$ chloral hydrate and

\section{Table 1}

\begin{tabular}{lll} 
& Runner & Nonrunner \\
\hline Number of investigated sections & 3 & 3 \\
Average no. of sampling sites & $8-10$ & $8-10$ \\
Counting frame width $(\mathrm{X})(\mu \mathrm{m})$ & 50 & 50 \\
Counting frame height $(\mathrm{Y})(\mu \mathrm{m})$ & 50 & 50 \\
Counting frame area $(\mathrm{XY})\left(\mu \mathrm{m}^{2}\right)$ & 2500 & 2500 \\
Sampling grid $(\mathrm{X})(\mu \mathrm{m})$ & 118 & 118 \\
Sampling grid $(\mathrm{Y})(\mu \mathrm{m})$ & 74 & 74 \\
Sampling grid area $(\mathrm{XY})\left(\mu \mathrm{m}^{2}\right)$ & 8732 & 8732 \\
Section periodicity & 10 & 10 \\
Area sampling fraction $(\mathrm{asf})$ & 3.5 & 3.5 \\
Average markers counted & 80 & 47 \\
Average estimated total by physical & 275 & 163 \\
$\quad$ dissector (HDB at 12 th week) & & $<0.2$ \\
Average estimated CE & $<0.2$ & \\
\hline
\end{tabular}


then perfused with ringers solution followed by $4 \%$ paraformaldehyde (PFA) in $0.1 \mathrm{M}$ phosphate buffer (PB) (pH 7.4). The brain was subsequently post-fixed for $4 \mathrm{~h}$ in $4 \%$ PFA $\left(4^{\circ} \mathrm{C}\right)$ and then in $0.1 \mathrm{M} \mathrm{PB}$ containing $15 \%$ sucrose overnight at $4{ }^{\circ} \mathrm{C}$. The next day, the specimens were embedded in Lipshaw M-1 embedding matrix before liquid nitrogen treatment. Frozen sections of $30 \mu \mathrm{m}, 2 \mathrm{~mm}$ from the tip of forebrain were cut, and every 10th section selected for mounting onto gelatinized slides. The dried slides were then washed with PBS-TX $(0.2 \%$ Triton-X) before they were blocked with the appropriate serum (5\%) for an hour. This was followed by overnight incubation with the polyclonal goat anti-rat choline acetyltransferase (ChAT) (1:100; Chemicon, Catalog no. AB144P). Subsequent antibody detection was carried out using the Vectastain ABC kit (Vector Laboratories, PK-4000) with 3,3'diaminobenzidine (DAB) (Sigma, Catalog no. D5637) as the peroxidase substrate.

\subsection{Stereologic analysis}

To estimate the number of cholinergic neuronal profile in the MSDB, a systematic random sampling series of sections starting from the level of the anterior commissure to the genu of the corpus callosum were taken for stereological analysis (Paxinos and Watson, 1998). The analysis was performed using a stereology workstation running StereoInvestigator (version 6.0, Microbrightfield). Quantification was estimated with the "Fractionator" probe (Schmitz and Hof, 2005) on photomicrographs taken previously of the various sections. All cell profiles in contact or within the inclusion zone of the "counting frames" were marked for counting. Other parameters associated with the procedure were listed accordingly in Table 1 . Subsequently, the software was able to estimate the average number of cholinergic neuronal profile in each subnucleus (MSN, VDB, HDB) in each animal. The collated results over the 3 time points were assembled and graphed as detailed in Fig. 8.

\subsection{Statistics}

All data collected were analyzed using the SPSS for Windows (Version 12). The escape latencies, pathlength, and swim speed in the water maze were analyzed by two-way ANOVA for between-subjects differences between runners and nonrunners ("RUNNING" effect) and repeated measures (within subjects) effects across block interval 1 to 3 ("BLOCK" effect). The probe trial data for percentage of time spent in each of the four zones were analyzed by multivariate ANOVA. For the stereological analysis, the independent sample $t$ test was applied to test for any differences between the runners and nonrunners. A $p<0.05$ is designated to be significant. All data are presented as mean $\pm \mathrm{SEM}$, calculated where appropriate from the ANOVA estimated marginal means and pooled error terms.

\section{Acknowledgments}

This project was supported by a research grant (R-181-000069-112) from the National University of Singapore (NUS). Eng-
Tat Ang was a recipient of the NUS postgraduate research scholarship during the project. We wish to thank Ms. Donna Tan for her technical advice concerning the water maze setup. The authors are also indebted to Dr. Patrick Gregory (Postdoctoral Fellow, University of Fribourg, Switzerland) for proof reading the manuscript.

\section{R E F E R E N C ES}

Abercrombie, M., 1946. Estimation of nuclear population from microtome sections. Anatomical Record 94, 239-247.

Abraham, W.C., 2003. How long will long-term potentiation last? Philosophical Transactions of the Royal Society of London. Series B, Biological Sciences 358, 735-744.

Anderson, B.J., Rapp, D.N., Baek, D.H., McCloskey, D.P., Coburn-Litvak, P.S., Robinson, J.K., 2000. Exercise influences spatial learning in the radial arm maze. Physiology and Behavior 70, 425-429.

Anderson, B.J., Eckburg, P.B., Relucio, K.I., 2002. Alterations in the thickness of motor cortical subregions after motor-skill learning and exercise. Learning and Memory 9, 1-9.

Ang, E.T., Wong, P.T., Moochhala, S., Ng, Y.K., 2003. Neuroprotection associated with running: is it a result of increased endogenous neurotrophic factors? Neuroscience 118, 335-345.

Ang, E.T., Wong, P.T., Moochhala, S., Ng, Y.K., 2004. Cytokine changes in the horizontal diagonal band of Broca in the septum after running and stroke: a correlation to glial activation. Neuroscience 129, 337-347.

Arida, R.M., Scorza, C.A., Da Silva, A.V., Scorza, F.A., Cavalheiro, E.A., 2004. Differential effects of spontaneous versus forced exercise in rats on the staining of parvalbumin-positive neurons in the hippocampal formation. Neuroscience Letters 364, 135-138.

Barnes, C.A., Forster, M.J., Fleshner, M., Ahanotu, E.N., Laudenslager, M.L., Mazzeo, R.S., Maier, S.F., Lal, H., 1991. Exercise does not modify spatial memory, brain autoimmunity, or antibody response in aged F-344 rats. Neurobiology of Aging 12, 47-53.

Braszko, J.J., Kaminski, K.A., Hryszko, T., Jedynak, W., Brzosko, S., 2001. Diverse effects of prolonged physical training on learning of the delayed non-matching to sample by rats. Neuroscience Research 39, 79-84.

Butler, M.P., O'Connor, J.J., Moynagh, P.N., 2004. Dissection of tumor-necrosis factor-alpha inhibition of long-term potentiation (LTP) reveals a p38 mitogen-activated protein kinase-dependent mechanism which maps to early-but not late-phase LTP. Neuroscience 124, 319-326.

Christianson, S.-A. (Ed.), 1992. Handbook of Emotion and Memory: Current Research and Theory. Erlbaum Associates, Hillsdale, New Jersey.

Cummins, R.A., Walsh, R.N., Budtz-olsen, O.E., Konsiantinos, T., Horsfall, C.R., 1973. Environmentally-induced changes in the brains of elderly rats. Nature 243, 516-518.

Cunningham, A.J., Murray, C.A., O'Neill, L.A., Lynch, M.A., O'Connor, J.J., 1996. Interleukin-1 beta (IL-1 beta) and tumour necrosis factor (TNF) inhibit long-term potentiation in the rat dentate gyrus in vitro. Neuroscience Letters 203, 17-20.

Diesfeldt, H.F., Diesfeldt-Groenendijk, H., 1977. Improving cognitive performance in psychogeriatric patients: the influence of physical exercise. Age and Ageing 6, 58-64.

Emery, C.F., Huppert, F.F., Schein, R.L., 1995. Relationships among age, exercise, health and cognitive function in a British sample. Gerontologist 35, 378-385.

Fordyce, D.E., Farrar, R.P., 1991a. Physical activity effects on hippocampal and parietal cortical cholinergic function and 
spatial learning in F344 rats. Behavioral Brain Research 43, 115-123.

Fordyce, D.E., Farrar, R.P., 1991b. Enhancement of spatial learning in F344 rats by physical activity and related learning-associated alterations in hippocampal and cortical cholinergic functioning. Behavioural Brain Research 46, 123-133.

Frick, K.M., Price, D.L., Koliatsos, V.E., Markowska, A.L., 1997. The effects of nerve growth factor on spatial recent memory in aged rats persist after discontinuation of treatment. Journal of Neuroscience 17, 2543-2550.

Frick, K.M., Stillner, E.T., Berger-Sweeney, J., 2000. Mice are not little rats: species differences in a one-day water maze task. NeuroReport 11, 3461-3465.

Gold, P.E., 2003. Acetylcholine modulation of neural systems involved in learning and memory. Neurobiology of Learning and Memory 80, 194-210.

Gould, E., McEwen, B.S., Tanapat, P., Galea, L.A., Fuchs, E., 1997. Neurogenesis in the dentate gyrus of the adult tree shrew is regulated by psychosocial stress and NMDA receptor activation. Journal of Neuroscience 17, 2492-2498.

Markowska, A.L., Price, D., Koliatsos, V.E., 1996. Selective effects of nerve growth factor on spatial recent memory as assessed by a delayed nonmatching-to-position task in the water maze. Journal of Neuroscience 16, 3541-3548.

McEwen, B.S., 2000a. Effects of adverse experiences for brain structure and function. Biological Psychiatry 48, 721-731.

McEwen, B.S., 2000b. The neurobiology of stress: from serendipity to clinical relevance. Brain Research 886, 172-189.

McGaugh, J.L., 2000. Memory-A century of consolidation. Science 287, 248-251.

Narath, E., Skalicky, M., Viidik, A., 2001. Voluntary and forced exercise influence the survival and body composition of ageing male rats differently. Experimental Gerontology 36, 1699-1711.

Neeper, S.A., Gomez-Pinilla, F., Choi, J., Cotman, C.W., 1996. Physical activity increases mRNA for brain-derived neurotrophic factor and nerve growth factor in rat brain. Brain Research 726, 49-56.

Paxinos, G., Watson, C., 1998. The Rat Brain in Stereotaxic Coordinates, 4th ed. Academic Press, San Diego.

Samorajski, T., Delaney, C., Durham, L., Ordy, J.M., Johnson, J.A., Dunlap, W.P., 1985. Effect of exercise on longevity, body weight, locomotor performance, and passive-avoidance memory of C57BL/6J mice. Neurobiology of Aging 6, 17-24.

Schmitz, C., Hof, P.R., 2005. Design-based stereology in neuroscience. Neuroscience 130, 813-831.

Shen, H., Tong, L., Balazs, R., Cotman, C.W., 2001. Physical activity elicits sustained activation of the cyclic AMP response element-binding protein and mitogen-activated protein kinase in the rat hippocampus. Neuroscience 107, 219-229.

Toni, N., Buchs, P.A., Nikonenko, I., Bron, C.R., Muller, D., 1999. LTP promotes formation of multiple spine synapses between a single axon terminal and a dendrite. Nature 402, 421-425.

Van Praag, H., Christie, B.R., Sejnowski, T.J., Gage, F.H., 1999. Running enhances neurogenesis, learning, and long-term potentiation in mice. Proceedings of the National Academy of Sciences of the United States of America 96, 13427-13431.

Wyss, J.M., Chambless, B.D., Kadish, I., van Groen, T., 2000. Age-related decline in water maze learning and memory in rats: strain differences. Neurobiology of Aging 21, 671-681.

Young, R.J., 1979. The effect of regular exercise on cognitive functioning and personality. British Journal of Sports Medicine 13, 110-117. 